\title{
Erosion on Titan: Past and Present
}

\author{
Ralph D. Lorenz and Jonathan I. Lunine \\ Department of Planetary Sciences, Lunar and Planetary Laboratory, University of Arizona, Tucson, Arizona 85721 \\ E-mail: rlorenz@lpl.arizona.edu
}

Received June 26, 1995; revised December 21, 1995

We examine various mechanisms of erosion on Titan and their likely effect on Titan's geomorphology. Specifically we evaluate the solubility of likely crustal ices (water and ammonia) in liquid methane and ethane, and compare these with the solubility of rocks on Earth. Aeolian and diurnal weathering can be neglected, and glacial erosion is unlikely unless the atmosphere has frozen out in the past. Erosion due to rainfall is at most comparable to periglacial environments on Earth. We find that additional laboratory experiments are required if Cassini observations are to be adequately interpreted. $\odot 1996$ Academic Press, Inc.

\section{INTRODUCTION}

Titan presents a fascinating body in terms of comparative planetology, most particularly because its atmospheric pressure is similar to that of the Earth, and because liquids may exist on its surface. The Earth's surface, and to a much lesser extent, that of Mars, has been significantly modified by erosion, in particular that due to liquids. Erosion on Titan has been considered briefly by Lunine and Stevenson (1985) and Lorenz (1993a). Sagan and Dermott (1982) suggested that tidal erosion in hydrocarbon oceans would have removed most of any land masses on Titan. In this paper we examine quantitatively the strengths of erosion mechanisms on Titan.

We divide the discussion into three sections: first we consider the solubility of likely Titan surface materials in the fluids that may lie there, a topic that requires revision since it was last examined because of new experimental data. Second, we consider other, more "mechanical" modes of erosion. Finally, we discuss the likely effects of erosive processes on Titan's landscape and investigate the Cassini mission's capabilities for identifying and characterizing erosive features.

There is at present no direct information constraining the composition of Titan's surface, although radar data by Muhleman et al. (1995) suggest that at least part of the surface is solid. The solid material is most likely water ice, by analogy with the other satellites in the Saturnian system on which water ice has been positively identified. It is unlikely to be pure, although the nature, amount, and distribution of the contaminants (silicates, organics, and perhaps ammonia-water ice) are not known, but see Coustenis et al. (1995) for recent work. The presence of atmospheric methane (which has a short photolytic lifetime) is evidence, albeit circumstantial, of one or more reservoirs on or near the surface of liquid hydrocarbons. The data available at present are inadequate to confirm or deny the existence of such reservoirs, which could be seas, lakes, underground caverns, or "soaked" regolith.

We consider not only Titan's present atmosphere and climate conditions, but also three possible paleoclimates.

1. A cooler epoch, due to the faint early sun (see McKay et al. 1993).

2. A cold, thin atmosphere, where methane levels have fallen such that the atmospheric opacity has dropped and surface temperatures fallen, leading to partial atmospheric collapse (Lorenz et al. 1995). Essentially methane and hydrogen provide the opacity for a modest greenhouse effect: if methane is depleted, the greenhouse is less efficient. If temperatures fall to the point where nitrogen condenses, the combination of high ice albedo and the faint early sun may lead to freeze-out of most of the nitrogen in the atmosphere.

3. A warm, thick atmosphere, generated by enhanced surface heat flux (tidal, accretional) and surface reservoirs of volatiles such as nitrogen and methane: the collisioninduced opacity of the atmosphere and the strong pressure/ temperature dependence make the surface temperature quite sensitive to heat flow from the interior (McKay et al. 1993).

\section{SOLUTION EROSION}

This section may be usefully divided into the discussion of the solubility of "bedrock" materials on Titan (water ice and water-ammonia) and "organics." We assume that the latter form a superficial layer on Titan-they may be responsible for albedo variations, but not for significant topography, both because of their limited thickness (per- 
haps hundreds of meters) but most importantly because they have low or zero strength, having been deposited as aerosol particles. We do not consider here the solubility of silicates (which may be present in some abundance on Titan's surface) in hydrocarbons; we expect it to be quite low.

\section{Solubility of Water Ice and Ammonia Ice in Cryogenic Liquids}

Experimental work by Rebiai et al. (1983) indicated a surprisingly measurable solubility of water ice in nonpolar, cryogenic liquids; in methane at $112 \mathrm{~K}$ a mole fraction of water of $6 \times 10^{-5}$ was reported. However, these results have since been shown to be spurious (Rest et al. 1990), and more recent experiments can place only upper limits on the solubilities: $10^{-8}$ mole fraction of water in liquid nitrogen, oxygen, and argon (Rest et al. 1990). Hence for the present work we must estimate the solubilities using a thermodynamic model, and it is the purpose of this section to do so.

We use "regular" solution theory (Prausnitz 1969) to quantify the solubility of water ice and ammonia ice in liquid hydrocarbons. A regular solution has zero excess entropy of mixing at constant volume and constant temperature, where the excess entropy (divided by the gas constant $R$ ) is defined relative to the ideal entropy of mixing,

$$
\Delta S_{\text {mix,ideal }} / R=-n_{1} \log \frac{n_{1}}{n_{1}+n_{2}}-n_{2} \log \frac{n_{2}}{n_{1}+n_{2}},
$$

where $n_{x}$ is the number of molecules of species $x$ in the solution (Prausnitz 1969). While the excess entropy of mixing is zero in a regular solution, the excess enthalpy is in general nonzero.

Physically, an ideal liquid solution (one in which all excess thermodynamic quantities are zero) corresponds to a situation where interactions between the molecules in solution are all the same. In other words, given two types of molecules $\mathrm{A}$ and $\mathrm{B}$ in an ideal solution, interactions between two A molecules, between two B molecules, or between an A and a B molecule are fully identical. Such an idealization is approached by solutions whose component molecules are similar in size to each other and exhibit weak (or, more restrictedly, very similar) molecular interactions.

A regular liquid solution, on the other hand, is consistent with a physical model in which the nature of the molecular interactions differs for the two different components of the solution, but the components are distributed randomly. This latter restriction eliminates some highly structured liquid solutions from consideration as regular. In particular, very polar liquid solutions such as ammonia-water are not good candidates for treatment as regular solutions. However, for water or ammonia as dilute solutes in solu- tions of essentially nonpolar liquids, regular solution theory provides a reasonable physical description, and certainly a much better one than that afforded by assuming an ideal case.

In the Appendix we derive, based on Prausnitz (1969) and Preston and Prausnitz (1970), the solubility of minor species $x_{2}$ in a liquid based on regular solution theory,

$$
x_{2}=\exp \left\{\frac{\Delta H}{R}\left[\frac{1}{T_{\mathrm{m}}}-\frac{1}{T}\right]-\frac{\left(\delta_{1}-\delta_{2}\right)^{2}}{R T} v_{2}^{\mathrm{L}}\right\} \text {. }
$$

Here the solute, subscripted " 2 ," is either water or ammonia, $\Delta H$ is the enthalpy of melting of the solute at its pure melting temperature $T_{\mathrm{m}}$, and $v_{2}^{\mathrm{L}}$ is the molar volume of the pure solute cooled to the solution temperature $T$ (as a metastable liquid, since pure water and ammonia are solids at relevant temperatures). The $\delta$ 's are "solubility" parameters which characterize the nonideal behavior of the solute and solvent.

To determine the solubility parameters $\delta_{i}$ for methane and ammonia we use the results of a corresponding states model tabulated by Preston and Prausnitz (1970). For water, this approach is not available, and we use instead the relation $\delta_{2}=\sqrt{\Delta U_{2} / v_{2}^{\mathrm{L}}}$, where $\Delta U_{2}$ is the complete energy of vaporization of the subcooled liquid and $v_{2}^{\mathrm{L}}$ is the molar volume of the subcooled liquid. Extrapolation of the heat of vaporization and molar volume of the liquid down to low temperatures is not reliable; in any event the solubility of water in methane and ethane is so low that it suffices to use values for $\Delta U_{2}$ and $v_{2}^{\mathrm{L}}$ near the water freezing point. These will tend to yield overestimates of the solubility. For pure ammonia, we extrapolate the vaporization energy and molar volume down to low temperatures using the

TABLE I

Parameters Used in Solubility Calculation

\begin{tabular}{lrr}
\hline \multicolumn{1}{c}{ Property } & $\mathrm{H}_{2} \mathrm{O}$ & $\mathrm{NH}_{3}$ \\
\hline$\Delta H(\mathrm{cal} / \mathrm{mole})$ & 1440 & 1309 \\
$T_{m}(\mathrm{k})$ & 273 & 195 \\
$v_{2}^{L}\left(\mathrm{~cm}^{3} / \mathrm{mole}\right)$ & 18 & 21 \\
\hline & \multicolumn{2}{c}{$\delta_{2}-\delta_{1}\left(\mathrm{cal} / \mathrm{cm}^{3}\right)^{0.5}$} \\
\cline { 2 - 3 } & $94 \mathrm{~K}$ & $180 \mathrm{~K}$ \\
\hline & \multicolumn{2}{c}{16.5} \\
$\mathrm{H}_{2} \mathrm{O}$ in $\mathrm{CH}_{4}$ & 14.7 & 18.5 \\
$\mathrm{H}_{2} \mathrm{O}$ in $\mathrm{C}_{2} \mathrm{H}_{6}$ & $9.1-12.1$ & $9.6-11.4$ \\
$\mathrm{NH}_{3}$ in $\mathrm{CH}_{4}$ & $7.3-10.3$ & $9.0-10.3$ \\
$\mathrm{NH}_{3}$ in $\mathrm{C}_{2} \mathrm{H}_{6}$ & & \\
\hline
\end{tabular}

Note. Values derived from data in Preston and Prausnitz (1970), and Haar and Gallagher (1978); the range shown for $\delta_{1}-\delta_{2}$ for ammonia reflects the two models discussed in the text. 


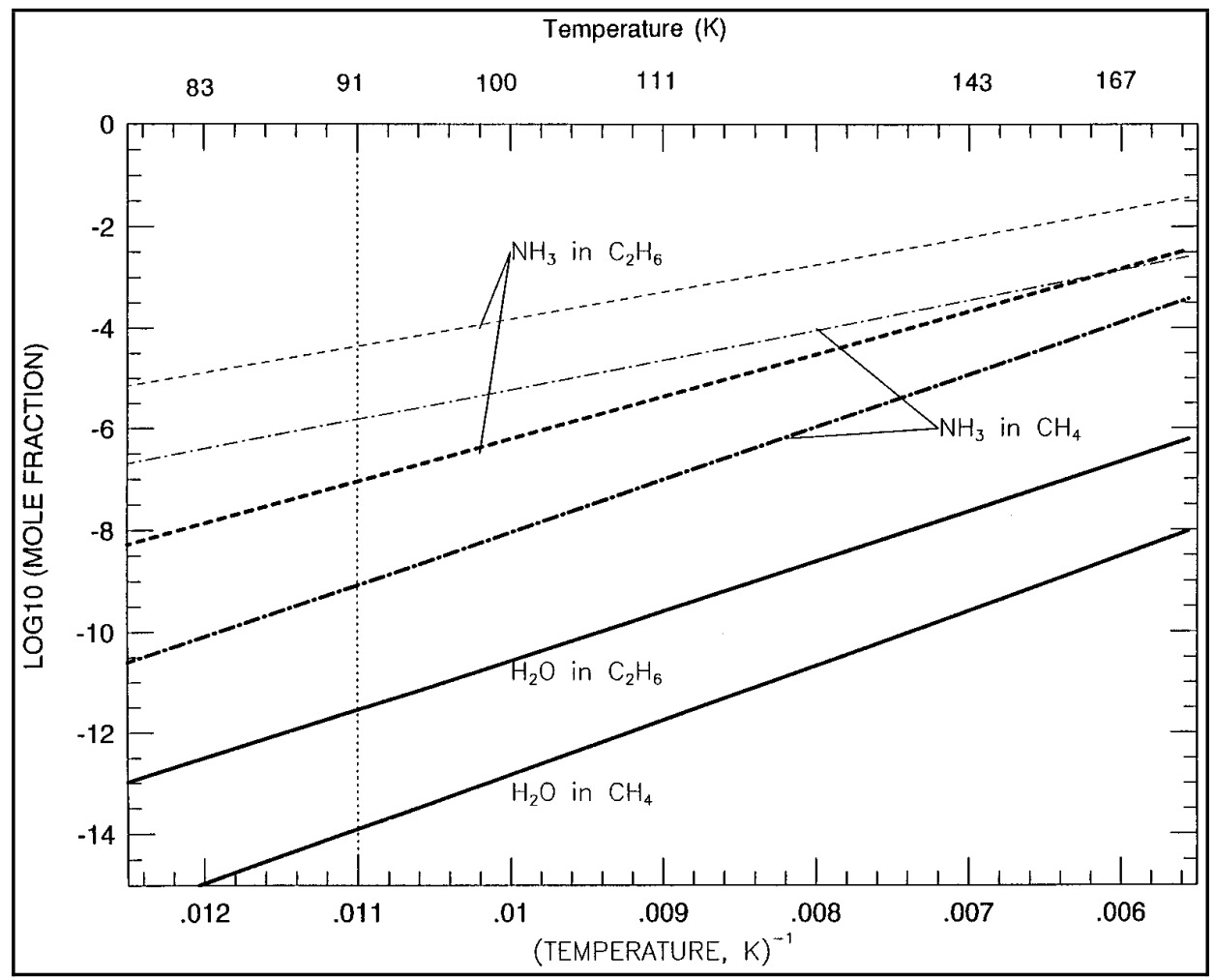

FIG. 1. Mole fraction of water and ammonia in liquid methane and ethane versus inverse temperature. For ammonia, the results of two approaches for computing the solubility parameters are shown for ethane (dashed) and methane (dot-dashed) as solvents: the thin lines are for the corresponding states tabulation of Preston and Prausnitz (1970), while the heavy lines are for the complete energy of vaporization estimate described in the text. For water as solute only the latter technique was applied. The vertical dotted line gives the approximate freezing points for pure methane and ethane; at lower temperatures liquid is stable only for mixtures of the two solvents. Parameter values are given in Table I.

tabulated thermodynamic properties of Haar and Gallagher (1978) as well as of Croft et al. (1988). Because the solubility of ammonia is not low we desire a second estimate of the solubility parameters, and we use the corresponding states estimate of Preston and Prausnitz (1970). Parameters used for the calculation are summarized in Table I.

Values of $\delta_{2}-\delta_{1}$ and resulting solubility were computed at 180 and $94 \mathrm{~K}$, near the extremes of the liquid field for pure methane and pure ethane. We then extrapolated the results down to lower temperatures using an Arrheniustype relation $x_{2}=\exp (-A / T+B)$, where $A$ and $B$ are constants. Below $91 \mathrm{~K}$ pure methane and ethane are solids, but on Titan mixtures of the two (as well as with nitrogen) will be liquid down to much lower temperatures. While we have not attempted to estimate the solubility for the case of mixed solvents the range of solubility between the methane and ethane provides a crude guide.

Figure 1 shows the results. The values for water are consistent with the experimental upper limits in Rest et al. (1990), which are for nitrogen, oxygen, and argon as the solvents; while direct comparison is not possible the experimental results serve as a guide since the variation due to nonpolar solvent type should be small. Of particular interest is the much higher predicted solubility of ammonia in the liquids. This suggests that crustal material containing ammonia may well be more susceptible to chemical erosion than pure water ice.

The two approaches for estimating solubility parameters (corresponding states versus energy of vaporization) for ammonia yield quite different results, particularly at low temperatures. Most uncertain is the goodness of the extrapolation of the vaporization energy $\Delta U_{2}$ to values well below the pure melting points of both water and ammonia. Near the ammonia melting point, on the right hand side of Fig. 1, the two approaches yield similar results; however, the divergence at lower temperatures indicates that the extrapolation may not be very good.

We do not a priori choose between the two solubility curves for ammonia as solute, preferring instead to regard the differences as a measure of the uncertainty of our theoretical approach. Two points should be made in this regard: (1) Solubilities in excess of $10^{-4}$ are probably unrealistic, because the solute is very polar and the solvent nonpolar (F. Raulin, personal communication, 1995); for the generally accepted value of Titan's surface temperature 
(95 K), and below, our solubilities do not exceed this. (2) The solubilities predicted for ammonia are large enough at $\sim 160 \mathrm{~K}$ that they can be measured using simple gravimetric experiments; such experiments are worth pursuing because of the implications of the results for erosion on Titan.

It may be noted that the higher solubility estimates for ammonia at Titan surface temperatures are similar in magnitude to the solubility of typical terrestrial minerals in water (e.g., calcite in water varies from 1 to $5 \times 10^{-4}$ mole/ liter or 0.2 to $1 \times 10^{-5}$ mole fraction, depending on the carbon dioxide concentration: silica is soluble to a similar degree (Krauskopf and Bird 1995)). For water ice in hydrocarbons, the solubilities we estimate are considerably lower than these values.

For an ammonia-water hydrate, for example, the monohydrate, the solubility may be intermediate between the ammonia and the water, though poor knowledge of the hydrate thermodynamic properties prevents a more detailed prediction. It should be noted that ammonia-water flows freezing at the temperature of Titan's surface (95 K) may form an amorphous solid (Yarger et al. 1993) in which the behavior of the ammonia could well be very different from that in the crystalline monohydrate; the pure ammonia value provides a reasonable upper limit to the solubility given these uncertainties.

\section{Hydrocarbon Solubilities}

Photochemical models (e.g., Yung et al. (1984), Lara et al. 1994, and Toublanc et al. 1995, plus erratum) predict the formation of a wide variety of organic compounds by methane photolysis: it is these organic compounds and their polymers that form the haze that renders Titan's atmosphere optically opaque. About $100 \mathrm{~m}$ of solid material may be produced over the age of the solar system, as well as a few hundred meters' worth of ethane.

The Preston-Prausnitz method may be applied to the solubility of other compounds in alkanes. SzczepaniekCieciak et al. (1978) applied the method to derive the solubility of a number of organic compounds in methane. It may be noted that the solubility ( $X$ mole fraction) of alkanes (see their Fig. 16) can be fitted by an equation of the form $\log _{10}(X)=0.5-0.83 n$, where $n$ is the number of carbon atoms in the chain.

In photochemical models of Titan's atmosphere, production rates for various compounds are determined. Table II shows the production rates of a number of compounds for the models by Yung et al. (1984), Lara et al. (1994), and Toublanc et al. (1995). The latter two (more recent) models predict similar ethane production rates less than half that of the Yung et al. (1984) model, and they predict considerably less propane. The predictions for acetylene production have considerable uncertainty, from factors of 4 to 1.3 less than the ethane production.
TABLE II Hydrocarbon Production Rates

\begin{tabular}{cccc}
\hline Compound & $\begin{array}{c}\text { Yung et al. } \\
(1984)\end{array}$ & $\begin{array}{c}\text { Lara et al. } \\
(1994)\end{array}$ & $\begin{array}{c}\text { Toublanc et al. } \\
(1995)\end{array}$ \\
\hline $\mathrm{C}_{2} \mathrm{H}_{6}$ & $5.8 \times 10^{9}$ & $2.3 \times 10^{9}$ & $2.6 \times 10^{9}$ \\
$\mathrm{C}_{2} \mathrm{H}_{2}$ & $1.2 \times 10^{9}$ & $5.6 \times 10^{8}$ & $1.9 \times 10^{9}$ \\
$\mathrm{C}_{3} \mathrm{H}_{8}$ & $3.8 \times 10^{8}$ & $1.4 \times 10^{6}$ & $5.1 \times 10^{6}$ \\
$\mathrm{C}_{4} \mathrm{H}_{10}$ & $2.3 \times 10^{7}$ & - & $6.2 \times 10^{7}$ \\
\hline
\end{tabular}

Note. Values are production rates in molecules $\mathrm{cm}^{-2} \mathrm{~s}^{-1}$.

The models as published do not list productions of individual compounds with carbon atom numbers larger than about 4 . However, these abundances can be estimated by the formula $A=A_{0} 10^{-\alpha\left(n-n_{0}\right)}$, suggested in Sagan and Thompson (1984) on the basis of laboratory simulations, where $\alpha$ is typically $0.25-1.0$ and $n_{0}$ is the number of carbon atoms in some reference compound. Taking $A_{0}$ as $3 \times 10^{9}$ molecules $/ \mathrm{cm}^{2} / \mathrm{sec}, n_{0}$ as 2 , multiplying by the age of the solar system $\left(1.4 \times 10^{17} \mathrm{sec}\right)$ and dividing by Avogadro's constant gives the total production of $10^{2.8-\alpha n+2 \alpha}$ moles per $\mathrm{cm}^{2}$. If $\alpha$ is 1 , then the deposited amount of each alkane is $10^{4.8-n}$ moles $/ \mathrm{cm}^{2}$. This may be compared with the solubility of alkanes in, say, $\sim 1000 \mathrm{moles} / \mathrm{cm}^{2}$ of methane (corresponding to about a 500-m depth), using the empirical solubility law mentioned above: the amount of each alkane that can be dissolved in this amount of methane is then $10^{3.5-0.83 n}$ moles $/ \mathrm{cm}^{2}$. For $n$ less than 8 , this is lower than the production value, so the ocean would become saturated and these short hydrocarbons would form seabed ("benthic") sediments.

If $\alpha$ is lower than 1 (as it may well be-see Fig. 6 in Sagan and Thompson (1984)) the picture is different. For $\alpha$ between about 0.85 and 1.00 , short hydrocarbons (with the critical $n$ value depending on $\alpha$ ) become saturated. If $\alpha$ is less than 0.85 , then the ocean can dissolve all alkanes completely without reaching saturation. This is probably the most likely scenario. However, restricting the discussion to alkanes dissolving in methane is not useful, as the range of ocean compositions on Titan is large, and the array of photochemical products is far more extensive than alkanes.

Raulin (1987) investigated the solubility of compounds in putative methane-ethane-nitrogen oceans in some detail-it may be seen from his figures that the solubility of most organics is rather higher (by $\sim$ two orders of magnitude) in ethane than in methane, and alkanes and alkenes do not saturate in the ocean. Dubouloz et al. (1989) reported the same conclusion and that alkynes and nitriles would be saturated in the ocean and form benthic sediments. These compounds would have saturation mole fractions of $10^{-4}$ to $10^{-7}$. Nitriles may be significant in altering 
the dielectric properties of surface liquids (Thompson and Squyres 1990).

For compounds such as alkynes and alkylnitriles which are not expected to dissolve in an ocean, their relative abundances in surface materials may be useful. Assuming that photochemical models can be adequately validated by terrestrial experiments and measurement of Titan's atmospheric composition, measurement of the abundance of these compounds in surface sediments may constrain their exposure history to solvents. While they do not reach saturation in the expected ocean, larger solvent volumes could leach them away. For example, the volume of methane recycled by geothermal heat flow could be about 100 times that of the ocean (Kossacki and Lorenz 1995), and the solvent volume leached by rainfall could be larger yet (see below).

The foregoing discussions are based on theoretical assessments of solubility of simple compounds. In reality the solvent will probably be a complex mixture of compounds, and the solutes will include complex heteropolymers ("tholins"). The laboratory data on these materials are at present scant indeed. McDonald et al. (1994) suggest that tholins are insoluble in hydrocarbons. However, this assertion is based on the observation that tholin does not dissolve in common organic solvents. The nondetection of dissolved tholin in these solvents, however, is based on the fact that solvents mixed with the tholins did not become discolored (B. N. Khare, personal communication, 1994). The corresponding solubility upper limits are of a similar order to the solubility levels described above. In any case, the complex tholins are likely to have a much lower abundance than other, simpler condensates. McKay (1995) reports an upper limit of tholin solubility in ethane of $0.03 \%$ by mass. Raulin (1987) made theoretical calculations of the solubility of organic polymers in methane and ethane and found that (except for polyethylene in methane) they would be practically insoluble.

\section{Chemical Erosion of a Topographic Feature}

The results of Fig. 1 can be used to estimate the erosive power of methane-ethane liquids moving over a fixed topograhic feature. Although remote sensing data argue for a mostly solid surface, they do not rule out lakes or small seas of hydrocarbon liquids (Muhleman et al. 1995, Lemmon et al. 1993, 1995, Griffith 1993, Coustenis et al. 1995, Smith et al. 1996). Assume for the moment a surface liquid layer which is undersaturated in water and ammonia. Then a crustal topographic feature of height and length $h$ composed of water ice or ammonia and submerged in the liquid will erode over a time scale given by Lunine and Stevenson (1985): $\delta t=1 / x_{2} \sqrt{h^{3} / D v}$, where $v$ is the velocity of the fluid moving over it, and $D$ is a diffusion coefficient. We use a typical $v=1 \mathrm{~cm} / \mathrm{sec}$ from tidal models (Sears 1995) and $D=10^{-4} \mathrm{~cm}^{2} / \mathrm{sec}$ from Lunine and Stevenson (1985). Then for a $0.1 \mathrm{~km}$ scale feature $t=3 / x_{2}$ years.

At $95 \mathrm{~K}$ solubilities of water are less than $10^{-11}$, and hence erosion exceeds the age of the solar system. For the much more soluble pure ammonia, however, erosion times from a few billion years down to a few tens of thousands of years are possible, depending on liquid composition and the solubility model chosen. Because ammonia is likely to be a relatively rare material at Titan's surface, hydrocarbon liquids may well remain undersaturated in the ammonia and thus erosion or leaching of features containing ammonia might occur. An important caveat is that the ammonia will be in the form of a hydrate with water, and the solubility of the ammonia in that configuration is likely to be lower.

\section{Exposure of Solutes to Solvents: Cycling}

A principal factor in assessing how much material can be removed by solution is the quantity of solvent to which the solute is exposed. One obvious case is where the total volume of the ocean is the appropriate solvent volume: consider a typical estimate of the globally averaged ocean depth of $\sim 500 \mathrm{~m}$. This corresponds to about $10^{7}$ moles/ $\mathrm{m}^{2}$. For a saturation mole fraction of $\mathrm{NH}_{3}$ in the ocean of $10^{-6}$, it follows that only about 10 moles $(170 \mathrm{~g})$ of ammonia can be dissolved per square meter of ocean, or over Titan's surface area of $8 \times 10^{13} \mathrm{~m}^{2}$, about a cube of sides $\sim 2 \mathrm{~km}$.

Thus, if ocean volume is used as a constraint, then there is unlikely to have been any significant solution erosion of topography.

However, if there is (or was) the methane equivalent of a hydrological cycle operating on Titan, then the solvent volume to which surface materials are exposed could be rather higher (indeed, by a factor of up to $\sim 10^{5}-$ see Rainfall Erosion below). Such a cycle relies, however, on the evaporation of methane at places, with the consequent deposition of solutes. The appropriate terrestrial analogues are desert playas and evaporite deposits.

At present, the surface of Titan is most likely isothermal, due to the thick atmosphere, so preferential evaporation of methane seems unlikely to be correlated with, for example, latitude. There may be a net downslope transport of solutes, due to the solution of surface deposits on mountains by rainfall which evaporates as it moves downhill. This effect may be enhanced by increased rainfall on higher terrain (Lorenz 1993b). Such a mechanism, which would operate more effectively on dark organic materials than on less soluble ices, may account for the large bright feature identified in HST images of Titan's surface (Smith et al., 1996).

As meteorological (i.e., solar-forced) solvent transport on a global scale seems unlikely even in previous epochs (i.e., an optically thin atmosphere, with appreciable equa- 
tor-to-pole temperature contrast, relies on an absence of methane), the most likely scenario for evaporation of solvents is geothermal activity. This might manifest itself both on the surface and in the subsurface.

In a porous regolith, hydrothermal circulation may lead to a geochemical "horizon" where solutes are redeposited: the circulation will be driven by the trickling of liquids down to warmer levels where they would evaporate, depositing solutes; the rising vapors would subsequently recondense and continue the washing down of solutes. On the surface, perhaps the most likely regions for deposition would be in crater basins - as well as topographic minima for the collection of liquids, these may penetrate into the crust and hence have a higher heat flow, leading to enhanced evaporation. An additional factor is that the impact event may itself deposit heat in the crust (in part as impact melt), heat which would take some time to be released (Thompson and Sagan 1992).

\section{MECHANICAL EROSION}

\section{Aeolian Erosion and Deposition}

On the Earth and Mars, there is significant erosion due to aeolian activity; specifically, surface particulates driven by wind can be strongly abrasive. Greeley and Iversen (1985) suggest that the erosion potential of windblown grains scales with the square of their velocity. Although the details depend on the trajectory of saltating grains, to first order the grain impact speed scales directly with the friction speed in the boundary layer. Work by Allison (1992) and Lorenz et al. (1995) suggests that friction velocities in Titan's boundary layer, and hence the velocities of wind-blown particles, are likely to be very small (of order $1 \mathrm{~cm} \mathrm{~s}^{-1}$ ).

The low friction velocities are due to the generally quiescent winds near the surface, due (as on Venus) to the large opacity of the atmosphere and the high density of the atmosphere near the surface. These factors first reduce the heat transport that the atmosphere must perform to balance seasonal and diurnal variations in insolation, and second, reduce the flow velocities required to effect that heat transport. As a counterexample, Mars has an atmosphere thin in density and optical terms, and has high wind speeds and consequently significant aeolian activity. This comparison is treated more fully in Lorenz et al. (1995).

We note also the recently published results of Hourdin et al. (1995), who have used a global circulation model to study Titan's winds. The model reproduces the strong superrotation at high altitudes (inferred from Voyager infrared data (Flasar et al. 1981) and the occultation by Titan of $28 \mathrm{Sgr}$ ) but suggests very low wind speeds near the surface, on the order of $1 \mathrm{~m} \mathrm{~s}^{-1}$ or less. The friction velocity (a measure of shear stress in the boundary layer) is typically a factor $C_{\mathrm{d}}^{0.5}$ lower than this, where $C_{\mathrm{d}}$ is the drag coefficient, typically 0.002 .

This low friction velocity (approximately two orders of magnitude lower than that of Earth or Mars) suggests aeolian erosion should be some four orders of magnitude less -indeed perhaps less yet, if any "threshold" speed for erosion to occur is relevant. An additional factor is that the thicker Titan atmosphere tends to cushion colliding particles, such that impact is further attenuated compared with Mars or Earth.

Thus aeolian erosive features such as yardangs (see, e.g., Greeley and Iversen 1985) seem improbable on Titan.

The obliteration of surface relief by the deposition of materials transported by aeolian processes is important on Mars. On Titan, however, it is unlikely to be significant, as the transport rate of aeolian materials is very small. This quantity scales as the cube of the friction velocity, so is approximately six orders of magnitude smaller on Titan than on Mars; a corollary of the low transport rate is the difficulty of forming dunes, which are not expected to be present on Titan (see Lorenz et al. 1995).

Lorenz et al. (1995) also considered possible alternative climate regimes on Titan, but found they are unlikely to have significantly higher transport rates.

\section{Glacial Erosion}

Large U-shaped valleys can be carved on Earth by the action of glaciers-gravity-driven flows of water ice. Ice glaciers may also have played a role on Mars (Kargel and Strom 1992).

As the underlying "bedrock" is water ice, with extremely low vapor pressure at Titan surface temperatures, an alternative precipitant must be sought for glacial erosion. The most obvious candidate is methane.

In this connection, the methane-nitrogen system is significant, since nitrogen tends to lower the liquidus temperature of methane. Solid methane-nitrogen can exist in the present atmosphere only at altitudes above $14 \mathrm{~km}$ (Kouvaris and Flasar 1991, Thompson et al. 1992)-methanenitrogen must be liquid or vapor at the surface. Thus, glaciers of solid methane seem improbable at present. Although the Voyager 1 radio-occultation measurement sampled near-surface temperatures only near the equator, the polar temperatures are not significantly cooler, due to the optical depth of the atmosphere, so in the present epoch, polar glaciers are similarly ruled out.

In earlier Titan atmospheres, however, it is possible that lower surface temperatures prevailed, allowing solid methane (more accurately, the methane-nitrogen mix) to exist on the surface (McKay et al. 1993). In such a case (case 1 ), however, the amount of material that could be deposited on the surface is small (say $\sim 5 \%$ of the atmosphere column mass, or about a 5-m depth of methane ice; as 
temperatures everywhere are low, the scope for mass transport and precipitation on particular areas is small). Such small amounts of material are inadequate to produce glacial features.

A further scenario (case 2) is that of the freeze-out of the atmosphere: this could arise if methane were depleted in the atmosphere by photolysis during intervals between episodic resupply (Lorenz et al. 1995) and the collisioninduced absorption of methane and hydrogen thereby vanished, allowing temperatures to fall and the atmosphere to freeze out.

Since, in such a freeze-out scenario, the atmospheric optical thickness would be small (except for the effects of nitrogen clouds), equator-to-pole temperature contrasts might be much higher than they are at present and deposition of nitrogen frost or snow might be expected to be higher at high latitudes. The $10^{5} \mathrm{~Pa}$ of the present atmosphere represents a column mass equivalent to about 100 $\mathrm{m}$ of nitrogen ice, if frozen globally. If deposited preferentially at high latitudes, the ice could be up to $\sim 1 \mathrm{~km}$ thick. As an analogy, consider the terrestrial ice ages, where ice sheets of order $1 \mathrm{~km}$ thick covered $30 \%$ of the Earth's surface (compared with ice over $10 \%$ of the surface today) and were associated with a global fall in sea level of about $100 \mathrm{~m}$ (Flint and Skinner 1974).

Detailed consideration of the rheology and pressuremelting effects of nitrogen glaciers is beyond the scope of this paper (see Brown and Kirk (1994) for a discussion of the influence of geothermal heat flow on Triton's polar caps: their expression for viscosity of nitrogen ice at the relevant temperatures gives values broadly similar to those for water ice in terrestrial environments). As a very rough comparison, consider that erosion rates on terrestrial glaciers can be expressed as proportional to the basal sliding velocity to the power of 1 or 2 (see, e.g., Harbor 1992), and that basal sliding rates are proportional to some power of the basal shear stress. This may be considered proportional to the slope, and the weight per unit area: for Titan's gravity of $1.35 \mathrm{~m} \mathrm{~s}^{-2}$, erosion rates are about a factor of 10 lower than those on Earth (where they are typically 0.1-20 mm/year). Thus U-shaped valleys on Titan large enough to be visible to Cassini's imaging radar (i.e., width and depth of order $0.5-1 \mathrm{~km}$ ) probably indicate an episode of atmospheric freeze-out of duration 0.25-100 Myr.

\section{Diurnal Weathering}

On Earth, the repeated diurnal temperature cycling of surface rocks can lead to stresses (especially in combination with evaporation of water leading to salt crystal formation, or the freezing of water giving a positive volume change) which can cause the rocks to break up.

This phenomenon is particularly acute in combination with other forms of erosion (such as aeolian or chemical weathering). However, we may safely neglect it on Titan as the radiative time constant of the troposphere (Flasar et al. 1981) is $\sim 140$ years, much longer than the diurnal period of 16 days, so temperature variations on the surface may be expected to be very small. Diurnal weathering on Titan with a putative optically thin atmosphere would be larger, perhaps, but the material being weathered would probably be nitrogen and other ices which have since melted or evaporated, so there would be no relics of such activity.

\section{Rainfall Erosion}

Rainfall is a principal agent of erosion on Earth, and perhaps also during the Noachian and Hesperian epochs on Mars (Craddock and Maxwell 1993). Work on modeling rainfall on present-day Titan (Lorenz 1993b) has suggested that even though raindrops can be larger on Titan than on Earth (9-mm diameter, rather than $6 \mathrm{~mm}$ ), since terminal velocities of raindrops are low in the low gravity and thick atmosphere of Titan, and since raindrops are likely to evaporate before they reach the surface, rainfall is not a strong agent of erosion on Titan. In this section we examine this question more explicitly.

Studies of soil erosion on Earth have been extensive, owing to the impact of erosion on agriculture. However, the expressions generated for estimating runoff are extremely empirical in nature. Zachar (1982) considers two methods gathered from the literature: the first, due to Ellison (1952), is of the form

$$
s \sim v^{4.33} d^{1.07} i^{0.65}
$$

where $s$ is the mass of soil splashed in $30 \mathrm{~min}, v$ is the raindrop velocity in $\mathrm{ft} / \mathrm{sec}, d$ is the drop diameter $(\mathrm{mm})$, and $i$ is the rainfall rate (in./hr). The empirical nature of this expression is evident from the rather mixed units. Since the constant of proportionality may be expected to be different for Titan in any case, we consider here only the terms and their exponents.

The strong dependence of erosion rate on impact velocity is clear. Another expression given in Zachar (1982) is due to Mirtskhulava (1970) and relates the erosion rate to $d^{1} v^{3}$, as long as $v$ exceeds a critical value $v_{\mathrm{c}}$ given by

$$
v_{\mathrm{c}}=(0.7 g M C / \varrho)^{0.5},
$$

where $g$ is the local gravity, $M$ is the coefficient of friction $(\sim 0.3), C$ is the cohesion of saturated soil $\left(0.05 \mathrm{~kg} \mathrm{~cm}^{-1}\right.$, although probably different on Titan), and $\varrho$ is the density of water. This expression gives $\sim 1 \mathrm{~m} \mathrm{~s}^{-1}$ for raindrops on Earth, or $\sim 1.77 \mathrm{~m} \mathrm{~s}^{-1}$ on Titan.

Lorenz (1993b) showed that the maximum velocity attainable by methane raindrops in the present Titan atmo- 
sphere is $\sim 1.6 \mathrm{~m} \mathrm{~s}^{-1}$, so if the same value of cohesion is assumed, rainfall should not cause erosion on Titan today. For a downpour consisting of the largest (fastest, most destructive drops) then Eq. (4) above gives an erosion rate ratio (Earth/Titan) for the same rainfall intensity of $(9.2 /$ $1.6)^{4.33}(6 / 9)^{1.07} \sim 1260$.

We can, additionally, constrain the global average rainfall rate. About 1\% of Titan's insolation is transported upward as a convective flux (McKay et al. 1992), or 0.035 $\mathrm{W} \mathrm{m}{ }^{-2}$. Taking the latent heat of vaporization of methane as $\sim 5 \times 10^{4} \mathrm{~J} \mathrm{~kg}^{-1}$, this leads to an upper limit of $7 \times 10^{-7}$ $\mathrm{kg} \mathrm{s}^{-1} \mathrm{~m}^{-2}$, or $\sim 4 \mathrm{~cm} /$ year. (The corresponding calculation for the Earth yields, assuming an average convective flux of $100 \mathrm{~W} \mathrm{~m}^{-2}$ (McIlveen 1992) and a specific heat of vaporization of $2.5 \mathrm{MJ} \mathrm{kg}^{-1}$, about $4 \times 10^{-5} \mathrm{~kg} \mathrm{~m}^{-2} \mathrm{~s}^{-1}$, or $\sim 1.2$ $\mathrm{m}$, which compares well with the estimated global annual precipitation of $1 \mathrm{~m}$ (McIlveen 1992)). On average, then, we may expect rainfall (the intensity-time product) to be $\sim 20$ times lower on Titan than on Earth.

Thus, to a first order, we can say that the erosive potential of rainfall on soils on Titan is $\sim 10^{4}$ times less than that on Earth. But note that in terms of solute transport, the equivalent of a $10^{8}$-m deep methane ocean could have been cycled through rainfall.

Alternatively, a larger-scale approach to the problem may be considered. Flint and Skinner (1974) note that of the $\sim 76 \mathrm{~cm}$ of average annual rainfall on the USA, about 50 $\mathrm{cm}$ evaporates, leaving $\sim 25 \mathrm{~cm}$ as runoff, with a potential energy of $\sim 10^{4} \mathrm{~J} \mathrm{~kg}^{-1}$. This leads to an erosion rate of about $6 \mathrm{~cm}$ per 1000 years.

If erosion on Titan scales with rain mass flux alone, then there is a factor $\sim 60$ less erosion rate on Titan. If, more realistically, the principal factor is the potential energy of rainfall, then assuming the same average altitude drop (from raindrop impact, until the liquid reaches the ocean or evaporates) for the rainfall $(\sim 1 \mathrm{~km})$, the factor is $\sim 400$ (taking into account Titan's lower gravity). These factors assume that all the rain on Titan is available as runoff. When the modest present-day methane humidity on Titan ( $>60 \%$ ) is considered, a large fraction will evaporate soon after hitting the ground, if indeed rain does reach the surface, so this is probably a strong overestimate of the erosion.

Conservatively, then, for Titan's present atmosphere, we conclude that erosion rates due to rainfall are at best $\sim 10^{2}$ less than those on Earth, and possibly several orders of magnitude lower. This corresponds to an upper limit of about $1 \mathrm{~m}$ every $10^{6}$ years. This upper limit is essentially the same as the lower of the denudation rate estimates for ancient Mars (Craddock and Maxwell 1993) of $10^{-5}-5 \times$ $10^{-4} \mathrm{~cm} /$ year, derived from data on crater degradation in the martian highlands. Similar rates are obtained in terrestrial periglacial environments.

These arguments all apply to the present climate on
Titan. It is worth investigating whether alternative historical climates could have had higher erosion rates. In these different atmospheres, the effects on erosion may be first considered by the effects on raindrop size and descent velocity. Lorenz (1993b) cites a formula by Clift et al. (1978) which suggests that the maximum raindrop diameter $d_{\max }$ is independent of atmospheric properties, and may be written as

$$
d_{\max } \sim Z\left(\gamma / g \varrho_{1}\right)^{0.5}
$$

where $\gamma$ is the surface tension, $\varrho_{1}$ is the liquid density and $Z$ is a constant. Clift et al. (1978) suggest $Z=4$; Lorenz (1993b) suggests $Z=2$, which gives more appropriate results. In any case, the maximum drop size should be the same as on the present-day Titan, or $9.5 \mathrm{~mm}$ in diameter, according to Lorenz (1993b).

The terminal velocity of these drops is, however, sensitive to the atmospheric properties and is given by

$$
V_{\max } \sim 2\left(\varrho_{1} g \gamma / \varrho_{\mathrm{a}}^{2}\right)^{0.25}
$$

Raindrop terminal speeds and erosion potentials are given in Table III for four scenarios.

Moderately thinner (case 1) atmospheres could have terminal descent velocities perhaps twice that expected at present, leading to an order of magnitude higher erosion rate per drop. It is difficult to conclude anything about rain fluxes, as the convective heat flux is not known for these cases. However, if the humidity is much higher, drops are less likely to evaporate during descent.

The cold, thin case 2 atmosphere could have much higher terminal velocities for drops than those indicated in Table III if its density were less than $0.1 \mathrm{~kg} \mathrm{~m}^{-3}$, but the atmospheric collapse is due to (and occurs $\sim 10^{6}$ years after) the depletion of atmospheric methane, so methane raindrops would simply not be present.

A thick, warm (case 3) atmosphere with a high density has lower terminal velocities, so is likely to produce less (if any) erosion than at present. However, the convective heat flux and hence the potential amount of rainfall could be higher than the present day's. This is uncertain, and it is reasonable to expect that the low rain terminal velocities are the dominant factor, so overall rainfall erosion potential is small.

As discussed in Lorenz (1993b), in the present atmosphere, drops can reach the ground on Titan under conditions of $60 \%$ humidity or less only if the ground is elevated above the nominal surface of the $2575-\mathrm{km}$ radius. On such elevated terrain, we might expect the erosion rates discussed above. If, on the other hand, erosive features due to pluvial action were found over widespread low terrain, it may be reasonable to deduce that a past climate epoch 
TABLE III

Raindrop Erosion

\begin{tabular}{|c|c|c|c|c|}
\hline $\begin{array}{c}\text { Atmospheric } \\
\text { density }\left(\mathrm{kg} \mathrm{m}^{-3}\right)\end{array}$ & $\begin{array}{c}\text { Terminal } \\
\text { speed } \\
\left(\mathrm{m} \mathrm{s}^{-1}\right)\end{array}$ & $\begin{array}{l}\text { Drops } \\
\text { erosive? }\end{array}$ & $\begin{array}{c}\text { Rain } \\
\text { probable? }\end{array}$ & $\begin{array}{l}\text { Erosive } \\
\text { potential }\end{array}$ \\
\hline 0.1 (case 2 ) & 11.4 & Very & No methane & Not applicable \\
\hline $1.0($ case 1$)$ & 3.6 & Yes & $?$ & Higher than present? \\
\hline 5.4 (present) & 1.54 & Marginal & ? & Limits given in text \\
\hline 9.0 (case 3$)$ & 1.2 & No & ? & Lower than present \\
\hline
\end{tabular}

existed where rainfall was more frequent and/or erosive, suggesting the McKay et al. (1993) methane-rich cold case (case 1 above). (We stress widespread in the sense that it is possible for some regions to have been at higher altitudes in the past, yet topography may have relaxed such that what might appear for a small region as pluvial erosion at low altitude actually occurred at higher altitude: we might hope that there would be other geomorphological clues that this had occurred.)

This diagnostic is especially powerful for fluvial features, in that for a fluid to persist long enough to move downslope and cut a valley, the evaporation rate must be small compared with the delivery rate due to rainfall. The presence of fluvial incisive features is an indicator of fluvial erosion being large compared with "downslope diffusion" (see Rinaldo et al. 1995). Downslope diffusion may include raindrop erosion and runoff, as well as aeolian processes. Although, as indicated in the foregoing discussions, these are likely to exist at a very modest level, they are less sensitive to climate conditions than is the presence of rivers, so the existence of river-cut valleys is a strong indicator of a wetter climate. A particularly appropriate analogy might be that of the caliche-topped mesas of southern Nevada: on Titan similar structures could evolve where water-ice sediments might be topped by a more resistant layer where organic materials (mediated by occasional light rainfall) have cemented the sediments together (like calcrete on Earth). Stronger rainfall, however, would run off the edges and erode the margins, forming a mesa.

The discussions above were based upon the upper limit of a Titan surface methane humidity of $60 \%$, which in turn relied on the assumption of a saturation upper limit of methane abundance at the tropopause. A recent reanalysis of the Voyager 1 radio-occultation data by McKay et al. (1995), follows the suggestion of supersaturation of methane in Titan's upper troposphere by Voyager IRIS measurements (Courtin et al. 1995; Samuelson, personal communication, 1994). When the saturation limit at the tropopause is relaxed, the surface humidity becomes unconstrained. In this case, methane rivers are possible in the current epoch. However, as McKay et al. (1995) point out, supersaturation in the troposphere relies on condensa- tion being inhibited by kinetic barriers: it follows then that rainfall is infrequent, and hence erosion due to rainfall is low.

\section{DETECTING EROSION WITH CASSINI}

We have discussed the implications of various erosive processes on geomorphology: the prime source of data may well be the imaging radar on the Cassini orbiter. Light scattering in the atmosphere, even in the window regions through which surface features have been detected from the Hubble Space Telescope (Smith et al. 1996), is likely to be sufficiently strong to prevent shadow measurements or photoclinometry by optical instruments. Stereomapping by the imaging science subsystem or the visual and infrared mapping spectrometer may yield useful topographic information, however. The Huygens probe carries a descent imager (DISR) which will obtain clearer and higher-resolution images of Titan's surfaces as it descends through the atmosphere; however, this instrument will see only a small fraction of Titan's surface. The imaging radar will be unaffected by atmospheric effects and will give geomorphological information on 10-30\% of Titan's surface, of a quality similar to (but at spatial resolutions sightly lower than) the Magellan coverage of Venus.

As well as detecting erosive features directly, these instruments will investigate the crater population on Titan. Engel et al. (1995) investigated atmospheric breakup of projectiles on Titan and the effects of atmospheric history on the cratering distribution. Another input to the interpretation of the Titan's cratering record is the obliteration rate of craters on Titan. If the minimum diameter crater expected on Titan from ice/rock impactors is about $20 \mathrm{~km}$ (Engel et al. 1995) and the depth/diameter ratio of craters of this size on icy satellites is on the order of 0.1 (Schenk 1989), then about $2 \mathrm{~km}$ of deposited sediments is required to obscure the crater.

The erosion rates due to rainfall, for example, of $1 \mathrm{~m} /$ Myr or less, suggest that perhaps the smallest craters should be obliterated after $\sim 2$ Gyr. Larger craters $(>50 \mathrm{~km}$, say) are likely to be sufficiently unmodified by erosion to be visible. 
Measurement by the surface science package (SSP) on the Huygens probe of the mechanical properties of the surface material at the Huygens probe landing site (Lorenz 1994) may indicate whether the particle size distribution has been affected by fluvial sorting, and whether material has been compacted (by rainfall, for example). Measurement of the organic content of the surface materials by the GCMS will constrain the surface's solvent exposure; following an analysis of the survivability of the impact of the Huygens probe on Titan's surface (Lorenz 1994), the probe gas chromatograph/mass spectrometer (GCMS) is being structurally reinforced to maximize the probability of its being able to make measurements of surface composition. In the event of a landing on a liquid, the GCMS will be able to measure directly the solvent and solute composition: the detectability limit for gas-phase measurements is $10^{-8}$ (Niemann et al. 1995), although sample acquisition and volatilization uncertainties on the surface may raise this threshold for surface materials. This value may be compared with the solubility mole fractions in Section 2. Further, the SSP may be able to constrain the depth of the liquid present using an acoustic sounder (Zarnecki 1992).

Thus, gross erosive features (such as glacial valleys) and the effects of erosion on the cratering record predicted in this paper should be detected by the instruments on the Cassini orbiter (radar, and perhaps optical imaging). The optical instruments will also indicate whether there is a correlation between albedo and topography, as would be expected by the influence of altitude on rainfall. The Huygens probe will examine more closely a small part of Titan's surface, and hopefully measure its composition and solvent exposure history.

\section{CONCLUSIONS}

We have examined the likely strengths of erosive processes on Titan and considered the effects of climate change on these processes. We conclude that:

1. Solution erosion is unlikely to remove significant water-ice topography on Titan; specifically, islands in a hydrocarbon ocean can be long-lived. Ammonia-rich ices are much more susceptible to erosion, but this may be limited by the saturation of the ocean.

2. Erosion due to rainfall is modest: at most it is comparable with periglacial environments on Earth, or on Mars during the late Noachian period. If it occurs at present, it occurs only at high elevations or latitudes.

3. Erosive processes at this level can nonetheless affect the cratering record. Disentangling the effects of erosion from the effects of atmospheric shielding (Engel et al. 1995) will be challenging.
4. Glacial features (presumably near the poles) would be strong indicators of an episode of atmospheric collapse on Titan, lasting on the order of $10^{6}$ years or more.

5. Aeolian erosion, and diurnal weathering, can be neglected on Titan.

6. Measurement of the composition of organic materials on the surface of Titan by the Huygens GCMS may be used to constrain the exposure of the surface materials to organic solvents, provided that terrestrial experiments can adequately determine the relative production rates of organic compounds on Titan and their solubility in liquid methane and ethane.

7. Compacted deposits of organics are probably indicative of evaporite deposits and indicate areas of preferential evaporation. Such areas are probably correlated solely with topography: there is not expected to be a significant correlation with latitude, even in previous climate epochs, although conceivably such deposits could be indicators of anomalously high geothermal heat flow.

8. Direct measurement of the solubility of substances, but especially nitriles, ammonia, and ammonia hydrates in methane and ethane, is required to confirm the theoretical predictions made in this paper and interpret Cassini/Huygens data.

\section{APPENDIX: DERIVATION OF THE REGULAR SOLUTION SOLUBILITY}

Consider equilibrium between solid and liquid phases in a system of two components, namely water or ammonia and some nonpolar liquid. The parameter used here which expresses the free energy content of component $i$ in a given phase $y$ is the fugacity (or generalized pressure) $f_{i}^{y}$,

$$
\frac{f_{i}^{y}}{f_{i}^{y}(0)}=\exp \frac{\left(\mu_{i}^{y}-\mu_{i}^{y}(0)\right)}{R T} .
$$

Here $\mu_{i}^{y}$ is the chemical potential of species $i$ in phase $y, R$ is the universal gas constant, and $T$ is the temperature; 0 indicates the reference conditions. For a pure ideal gas the fugacity reduces to the pressure.

The conditions for chemical equilibrium between the liquid (l) and solid phases (s) is then

$$
\gamma_{1}^{\mathrm{s}} x_{1}^{\mathrm{s}} f_{1}^{\mathrm{s}}=\gamma_{1}^{1} x_{1}^{1} f_{1}^{1} \gamma_{2}^{\mathrm{s}} x_{2}^{\mathrm{s}} f_{2}^{\mathrm{s}}=\gamma_{2}^{1} x_{2}^{1} f_{2}^{1}
$$

where $\gamma$, the activity coefficient, is defined so that $f_{i}^{1}=\gamma_{i}^{1} x_{i}^{1} f_{i}^{0}$. The fugacity in some (usually pure) standard state is $f_{i}^{0}$. Let component 1 be the nonpolar material which forms the liquid solvent, and component 2 the water ice or ammonia ice. We will assume that there is no solid solution phase; that is, $\gamma_{2}^{\mathrm{s}}=x_{2}^{\mathrm{s}}=1$. This is not strictly correct, in particular because a clathrate hydrate may form in the case where the solid phase is water ice. However, the kinetics of clathrate formation are so slow below 150 K (Bar-Nun et al. 1988) that such formation will be irrelevant on the time scale over which the liquid solution establishes equilibrium. (A thin rind of clathrate might form quickly, taking up a small amount of 
component 1 , but the uptake will very rapidly saturate and slow to a negligible rate (Barrer and Ruzicka 1962).)

We now specify the activity coefficient for component 2 in the liquid as

$$
x_{2} \gamma_{2}=f_{2}^{\mathrm{s}} / f_{2}^{\mathrm{L}},
$$

where we have eliminated redundant use of the liquid superscript 1 , given the specifications in the previous paragraph for the solid phase. The superscript $\mathrm{L}$ refers to the pure, subcooled liquid phase of component 2 under its own saturation vapor pressure at the temperature of the solution we are considering. It is a fictitious state, because the system under consideration is well below the freezing point of either water or ammonia, but this device will enable us to derive a simple expression for the solubility.

From Prausnitz (1969) we can now derive the ratio $f_{2}^{\mathrm{s}} / f_{2}^{\mathrm{L}}$ by running a thermodynamic cycle for the pure solid up to the melting point, across to the pure liquid, and back down to the temperature of interest $T$,

$$
\begin{aligned}
\log \frac{f_{2}^{\mathrm{s}}}{f_{2}^{\mathrm{L}}}= & \frac{\Delta H}{R}\left(\frac{1}{T_{\mathrm{m}}}-\frac{1}{T}\right)+\frac{\Delta c_{\mathrm{p}}}{R}\left(\frac{T_{\mathrm{m}}}{T}-1\right) \\
& -\frac{\Delta c_{\mathrm{p}}}{R} \log \frac{T_{\mathrm{m}}}{T}
\end{aligned}
$$

where $\Delta H$ is the enthalpy of melting of component 2 at its pure melting temperature $T_{\mathrm{m}}$, and $\Delta c_{\mathrm{p}}$ is the difference in heat capacity (constant pressure) between the pure (subcooled) liquid and solid phases of component 2 , this difference being assumed to be constant with temperatures between $T_{\mathrm{m}}$ and $T$.

The second and third terms on the right-hand side of Eq. (A4) roughly cancel. Substituting from Eq. (A3) then gives

$$
\log x_{2} \gamma_{2}=\frac{\Delta H}{R}\left(\frac{1}{T_{\mathrm{m}}}-\frac{1}{T}\right)
$$

It remains to specify $\gamma_{2}$, which accounts for the nonideal interactions between components 1 and 2 in the liquid solution. One model for the activity coefficient in a regular solution is known as the Scatchard-Hildebrand equation, one form of which is (Preston and Prausnitz 1970)

$$
\log \gamma_{2}=\frac{v_{2} \phi_{1}^{2}}{R T}\left[\left(\delta_{1}-\delta_{2}\right)^{2}+2 \lambda_{12} \delta_{1} \delta_{2}\right]
$$

where $v_{2}$ is the volume per mole of component 2 in solution, $\delta_{i}$ is the solubility parameter for component $i$ (to be defined in a moment), $\lambda_{12}$ is a constant describing the interaction between molecules of components 1 and 2 in solution, and $\phi_{1}$ is the volume fraction of component 1 (the solvent) in the solution,

$$
\phi_{1}=\frac{\left(1-x_{2}\right) v_{1}^{1}}{\left(1-x_{2}\right) v_{1}^{1}+x_{2} v_{2}^{\mathrm{L}}} .
$$

The solubility parameters are $\delta_{i}=\sqrt{\Delta U_{i} / v_{i}}$, where $\Delta U_{i}$ is the energy of vaporization of pure component $i$ per unit volume, at the temperature of interest (recalling that, for component 2, the subcooled liquid is the relevant phase). Note further that while $\delta_{1}, \delta_{2}$ are functions of temperature, for many substances $\delta_{1}-\delta_{2}$ is roughly constant with temperature.
We assume that $\lambda_{12}$ is small, in part because the $\delta_{2}$ values used here already have incorporated effects associated with mixing of water and ammonia in other, nonpolar, species. Hence the last term in Eq. (A6) is neglected. Combining Eqs. (A5), (A6), and (A7), we have the following expression for the mole fraction of ice or ammonia dissolved in nonpolar liquids:

$$
\begin{aligned}
x_{2}= & \exp \left\{\frac{\Delta H}{R}\left[\frac{1}{T_{\mathrm{m}}}-\frac{1}{T}\right]\right. \\
& \left.-\frac{\left(\delta_{1}-\delta_{2}\right)^{2}}{R T} \frac{v_{2}^{\mathrm{L}}\left[v_{1}^{1}\left(1-x_{2}\right)\right]^{2}}{\left[v_{1}^{1}+\left(v_{2}^{\mathrm{L}}-v_{1}^{1}\right) x_{2}\right]^{2}}\right\} .
\end{aligned}
$$

It will turn out that $x_{2} \ll 1$; under this condition Eq. (A8) becomes

$$
x_{2}=\exp \left\{\frac{\Delta H}{R}\left[\frac{1}{T_{\mathrm{m}}}-\frac{1}{T}\right]-\frac{\left(\delta_{1}-\delta_{2}\right)^{2}}{R T} v_{2}^{\mathrm{L}}\right\} .
$$

\section{ACKNOWLEDGMENTS}

R.D.L acknowledges a useful discussion with B. N. Khare of Cornell and with Tony Rest of the University of Southampton, UK. Francois Raulin and an anonymous reviewer are thanked for comments. J.I.L. was supported through the NASA Planetary Atmospheres and Geology/ Geophysics programs, and R.D.L through the Cassini project.

\section{REFERENCES}

Allison, M. A. 1992. A preliminary assessment of the Titan boundary layer. In Proceedings, Symposium on Titan, Toulouse, France, Oct. 1991, ESA SP-338, pp. 113-118.

Bar-Nun, A., I. Kleinfeld, And E. Kochavi 1988. Trapping of gas mixtures by amorphous water ice. Phys. Rev. B. 38, 7749-7754.

BARrer, R.M., AND A. RuzICKA 1962. Non stoichiometric clathrate compounds of water. Part 4. Kinetics of formation of clathrate phases. Trans. Faraday Soc. 58, 1962.

Brown, R. H., AND R. L. KIRK 1994. Coupling of volatile transport and internal heat flow on Triton. J. Geophys. Res. 99, 1965-1981.

Clift, R., R. J. Grace, and W. E. Weber 1978. Bubbles, Drops and Particles. Academic Press, New York.

Courtin, R., D. Gautier, And C. P. McKay 1995. Titan's thermal emission spectrum: Re-analysis of the Voyager infrared measurements. Icarus 114, 144-162.

Coustenis, A., E. Lellouch, J. P. Maillard, and C. P. McKay 1995. Titan's surface: Composition and variability from the near infrared albedo. Icarus 118, 87-104.

Craddock, R. A., ANd T. A. Maxwell 1993. Geomorphic evolution of the martian highlands through ancient fluvial processes. J. Geophys. Res. 98, 3453-3468.

Croft, S. K., J. I. Lunine, And J. S. Kargel 1988. Equation of state of water-ammonia liquid: Derivation and planetological applications. Icarus 73, 279-293.

Dubouloz, N., F. Raulin, E. Lellouch, and D. Gautier 1989. Titan's hypothesized ocean properties: The influence of surface temperature and atmospheric composition uncertainties. Icarus 82, 81-96.

Ellison, W. D. 1952. Raindrop energy and soil erosion. Agriculture (Oxford) 20, 78. 
Engel, S., J. I. Lunine, And W. K. Hartmann 1995. Cratering on Titan and implications for Titan's atmospheric history. Planet. Space Sci. 43, 1059-1066.

Flasar, F. M. 1983. Oceans on Titan. Science 221, 55-57.

Flasar, F. M., R. E. Samuelson, And B. J. Conrath 1981. Titan's atmosphere: Temperature and dynamics. Nature 292, 693-698.

Flint, R. F., And B. J. Skinner 1974. Physical Geology. Wiley, New York.

Greeley, R., And J. D. Iversen 1985. Wind as a Geological Process. Cambridge Univ. Press, Cambridge, England.

GrIfFITH, C. A. 1993. Evidence for surface heterogeneity on Titan. Nature 364, 511-514.

HaAr, L., And J. S. Gallagher 1978. Thermodynamic properties of ammonia. J. Phys. Chem. Ref. Data 7, 635-792.

Harbor, J. M. 1992. Numerical modeling of the development of U-shaped valleys by glacial erosion. Geol. Soc. Am. Bull. 104, 13641375.

Hourdin, F., O. Talagrand, R. Sadourny, R. Courtin, D. Gautier, AND C. P. MCKAY 1995. Numerical simulation of the general circulation of the atmosphere of Titan. Icarus 117, 358-374.

Kargel, J. S., And R. G. Strom 1992. Ancient glaciation on Mars. Geology 20, 3-7.

Khare, B. N., C. Sagan, E. T. Arakawa, F. Suits, T. A. Callcott, and M. W. Williams 1984. Optical constants of organic tholins produced in a simulated titanian atmosphere: From soft $\mathrm{x}$-ray to microwave frequencies. Icarus 60, 127-137.

Kossacki, K. J., AND R. D. Lorenz 1995. Hiding Titan's ocean: Densification and hydrocarbon storage in an icy regolith. Planet. Space Sci., in press.

Kouvaris, L. C., AND F. M. Flasar 1991. Phase equilibrium of methane and nitrogen at low temperatures: Application to Titan. Icarus 91, $112-124$

Krauskopf, K. B., AND D. K. BIRD 1995. Introduction to Geochemistry, 3rd ed. McGraw-Hill, New York.

Lara, L. M., R. D. Lorenz, And R. Rodrigo 1994. Liquids and solids on the surface of Titan. Planet. Space Sci. 42, 5-14.

Lemmon, M. T., E. Karkoschka, And M. Tomasko 1993. Titan's rotation: surface feature observed. Icarus 103, 329-332.

Lemmon, M. T., E. KarkoschKa, And M. Tomasko 1995. Titan's rotational light curve. Icarus 113, 27-38.

Lorenz, R. D. 1993a. The surface of Titan in the context of ESA's Huygens probe ESA J. 17, 275-292.

Lorenz, R. D. 1993b. The life, death and afterlife of a raindrop on Titan. Planet. Space Sci. 41, 647-655.

Lorenz, R. D. 1994. Huygens probe impact dynamics. ESA J. 18, 93-117.

Lorenz, R. D., J. I. Lunine, J. A. Grier, And M. A. Fisher 1995. Predicting aeolian activity on planets: Application to Titan paleoclimatology. J. Geophys. Res. 100, 26,377-26,386.

Lunine, J. I., AND D. J. STEVEnson 1985. Evolution of Titan's coupled ocean-atmosphere system and interaction of ocean with bedrock. In Ices in the Solar System (J. Klinger, D. Benest, A. Dollfus, and R. Smoluchowski, Eds.), pp. 741-757. Reidel, Dordrecht, The Netherlands.

McDonald, G. D., W. R. Thompson, M. Heinrich, B. N. Khare, and C. SAGAN 1994. Chemical investigation of Titan and Triton tholins. Icarus 108, 137-145.

MCIlveen, J. F. R. 1992, Fundamentals of Weather and Climate. Chapman and Hall, London.
McKAy, C. P. 1995. Composition and properties of Titan's organic haze. Planet. Space Sci., submitted.

McKay, C. P., J. B. Pollack, R. Courtin, and J. I. Lunine 1992. The atmospheric temperature structure of Titan. In Proceedings, Symposium on Titan, Toulouse, France, October 1991, ESA SP-338, pp. $77-80$.

McKay, C. P., J. B. Pollack, J. I. Lunine, and R. Courtin 1993. Coupled atmosphere-ocean models of Titan's past. Icarus 102, 88-98.

McKay, C. P., S. C. Martin, C. A. Griffith, and R. M. Keller 1995. Temperature lapse rate and methane in Titan's troposphere. Icarus, submitted.

Mirtskhulava, S. E. 1970. Engineering Methods of Calculation and Prognosis of Water Erosion (in Russian). Izd. Kolos, Moscow.

Muhleman, D. O., A. W. Grossman, B. J. Butler, and M. A. Slade 1995. Radar investigations of Mars, Mercury and Titan. Annu. Rev. Earth Planet. Sci. 23, 337-374.

Niemann, H., S. Atreya, K. Biemann, B. Block, G. Carignan, T. Doahue, L. Frost, D. Gautier, D. Harpold, D. Hunten, G. Israel, J. Lunine, K. Mauersperger, T. Owen, F. Raulin, J. RICHARDS, AND S. WAY 1995. The gas chromatograph mass spectrometer for the Huygens probe. ESA SP-1177, submitted.

Prausnitz, J. M. 1969. Molecular Thermodynamics of Fluid Phase Equilibria. Prentice Hall, Englewood Cliffs, NJ.

Preston, G. T., And J. M. Prausnitz 1970. Thermodynamics of solid solubility in cryogenic solvents. Ind. Eng. Chem. Process Des. Dev. 9, 264-271.

Raulin F. 1987. Organic chemistry in the oceans of Titan. Adv. Space Res. 7(5), 71-81.

Rebiai, R., A. J. Rest, and R. G. Scurlock 1983. The unexpectedly high solubility of water in cryogenic liquids. Nature 305, 412-413.

Rest, R. J., R. G. Scurlock, AND M. F. Wu 1990. The solubilities of nitrous oxide, carbon dioxide, aliphatic ethers and alcohols, and water in cryogenic liquids. Chem. Eng. J. 43, 25-31.

Rinaldo, A., W. E. Deitrich, R. Rigon, G. K. Vogel, and I. RodriGUEZ-ITURBE 1995. Geomorphological signatures of varying climate. Nature 374, 632-635.

Sagan, C., And S. F. Dermott 1982. The tide in the seas of Titan. Nature 300, 731-733.

SAGAN, C., AND W. R. ThOMPSON 1984. Production and condensation of organic gases in the atmosphere of Titan. Icarus 59, 133-161.

SCHENK, P. 1989. Crater formation and modification on the icy satellites of Uranus and Saturn: Depth/diameter and central peak occurrence. J. Geophys. Res. 94, 3813-3832.

SEARs, W. D. 1995. Tidal dissipation in oceans on Titan. Icarus 113, 39-56.

Smith, P. H., M. T. Lemmon, R. D. Lorenz, L. A. Sromovsky, J. J. Caldwell, ANd M. D. Allision 1996. Titan's surface, revealed by HST imaging. Icarus 119, 336-349.

Szczepaniec-Cieciak, E. B. Dabrowska, J. M. Lagan, and Z. WoJTASZEK 1978. Estimation of the solubility of solidified substances in liquid methane by the Preston-Prausnitz method. Cryogenics 18, 591-599.

Thompson, W. R., and C. Sagan 1992. Organic chemistry on TitanSurface interactions. In Proceedings, Symposium on Titan, Toulouse, France, October 1991, ESA SP-338, pp. 167-176.

Thompson, W. R., AND S. W. Souyres 1990. Titan and other icy satellites: Dielectric properties of constituent materials and implications for radar sounding. Icarus 86, 336-354.

Thompson, W. R., J. A. Zollweg, And D. H. Gabis 1992. Vapor-liquid equilibrium thermodynamics of $\mathrm{N}_{2}+\mathrm{CH}_{4}$ : Model and Titan applications. Icarus 97, 187-199. 
Toublanc, D., J. P. Parisot, J. Brillet, D. Gautier, F. Raulin, and C. P. MCKAY 1995. Photochemical modeling of Titan's atmosphere. Icarus 113, 2-26 (see also erratum in press).

YARGer, J., J. I. LunINE, AND M. F. BuRKe 1993. Calorimetric studies of the ammonia-water system with application to the outer solar system. $J$. Geophys. Res. 98, 13,109-13,117.
Yung, Y. L., M. Allen, And J. P. Pinto 1984. Photochemistry of the atmosphere of Titan : Comparison between model and observations. Astrophys. J. Suppl. 55, 465-506.

ZaChar, D. 1982. Soil Erosion. Elsevier/North-Holland, New York.

ZARNECKI, J. C. 1992. Surface science package for the Huygens probe. J. Br. Interplanet. Soc. 45, 365-370. 